\title{
Strategy to improve the accuracy of convolutional neural network architectures applied to digital image steganalysis in the spatial domain
}

Reinel Tabares-Soto ${ }^{\text {Corresp., } 1}$, Harold Brayan Arteaga-Arteaga ${ }^{1}$ Alejandro Mora-Rubio ${ }^{1}$ Mario Bravo ortiz ${ }^{1}$, Daniel Arias-Garzón ${ }^{1}$, Jesús Alejandro Alzate Grisales ${ }^{1}$, Alejandro Burbano Jacome ${ }^{1}$, Simon Orozco-Arias ${ }^{2,3}$, Gustavo Isaza $^{3}$, Raul Ramos Pollan ${ }^{4}$

1 Department of Electronics and Automation, Universidad Autonóma de Manizales, Manizales, Caldas, Colombia

2 Department of Computer Science, Universidad Autonoma de Manizales, Manizales, Colombia

3 Department of Systems and Informatics, Universidad de Caldas, Manizales, Colombia

4 Department of Systems Engineering, Universidad de Antioquia, Medellín, Antioquia, Colombia

Corresponding Author: Reinel Tabares-Soto

Email address: rtabares@autonoma.edu.co

In recent years, Deep Learning (DL) techniques applied to steganalysis have surpassed the traditional two-stage approach by unifying feature extraction and classification in a single model, the Convolutional Neural Network (CNN). Several CNN architectures have been proposed to solve this task, improving steganographic images' detection accuracy, but it is unclear which computational elements are relevant. Here we present a strategy to improve accuracy, convergence, and stability during training. The strategy involves a preprocessing stage with Spatial Rich Models (SRM) filters, Spatial Dropout, Absolute Value layer, and Batch Normalization. Using the strategy improves the performance of three steganalysis and two image classification CNN's, by enhancing the accuracy from $2 \%$ up to $10 \%$ while reducing the training time to less than 6 hours and improving the networks' stability. 


\title{
Strategy to improve the accuracy of convolutional neural network architectures applied to digital image steganalysis in the spatial domain
}

\author{
Reinel Tabares-Soto ${ }^{1}$, Harold Brayan Arteaga-Arteaga ${ }^{1}$, Alejandro \\ Mora-Rubio ${ }^{1}$, Mario Alejandro Bravo-Ortiz ${ }^{1}$, Daniel Arias-Garzón ${ }^{1}$, Jesus \\ Alejandro Alzate-Grisales ${ }^{1}$, Alejandro Buenaventura Burbano-Jacome ${ }^{1}$, \\ Simon Orozco-Arias ${ }^{2,3}$, Gustavo Isaza ${ }^{3}$, and Raúl Ramos-Pollán ${ }^{4}$ \\ ${ }^{1}$ Department of Electronics and Automation, Universidad Autónoma de Manizales, \\ Manizales, Colombia \\ ${ }^{2}$ Department of Computer Science, Universidad Autónoma de Manizales, Manizales, \\ Colombia \\ ${ }^{3}$ Department of Systems and Informatics, Universidad de Caldas, Manizales, Colombia \\ ${ }^{4}$ Department of Systems Engineering, Universidad de Antioquia, Medellín, Colombia \\ Corresponding author: \\ Reinel Tabares-Soto ${ }^{1}$ \\ Email address: rtabares@autonoma.edu.co
}

\begin{abstract}
In recent years, Deep Learning (DL) techniques applied to steganalysis have surpassed the traditional two-stage approach by unifying feature extraction and classification in a single model, the Convolutional Neural Network (CNN). Several CNN architectures have been proposed to solve this task, improving steganographic images' detection accuracy, but it is unclear which computational elements are relevant. Here we present a strategy to improve accuracy, convergence, and stability during training. The strategy involves a preprocessing stage with Spatial Rich Models (SRM) filters, Spatial Dropout, Absolute Value layer, and Batch Normalization. Using the strategy improves the performance of three steganalysis and two image classification CNN's, by enhancing the accuracy from $2 \%$ up to $10 \%$ while reducing the training time to less than 6 hours and improving the networks' stability.
\end{abstract}

\section{INTRODUCTION}

Steganography and steganalysis are two research fields related to hidden messages in digital multimedia files. The first one consists of hiding information and the second one on detecting whether a file has a message or not. The steganographic process is illustrated by the famous Prisoner Problem (Simmons, 1984), which presents a prison scenario where two prisoners try to exchange messages reviewed by the prison director, who decides whether to deliver the message content. Steganography referring to images can be applied in the spatial or frequency domain. In the spatial field, the algorithms change the value of some pixels on the image, making imperceptible to the human eye, for instance, the Least Significant Bits (LSB) of each pixel (Johnson and Jajodia, 1998; Fridrich et al., 2001). Some of the algorithms in this domain are HUGO (Pevny et al., 2010b), HILL (Li et al., 2014), MiPOD (Sedighi et al., 2016), S-UNIWARD (Holub et al., 2014), and WOW (Holub and Fridrich, 2012). On the frequency domain, steganography use several transformations, such as Discrete Cosine Transform (DCT), Discrete Wavelet Transform (DWT), and Singular Value Decomposition (SVD) (Tabares-Soto et al., 2020). The compression format Joint Photographic Experts Group (JPEG) is the most common and based on DCT, whose coefficients can be modified to include a hidden message. Some of the algorithms in this domain are J-UNIWARD (Holub et al., 2014), F5 (Westfeld, 2001), UED (Guo et al., 2014), and UERD30 (Guo

PeerJ Comput. Sci. reviewing PDF | (CS-2020:11:55117:1:1:NEW 26 Feb 2021) 
et al., 2015).

Steganalysis consists of detecting whether an image has a hidden message, is divided into two stages. Stage one consists of feature extraction (e.g., Rich Models (RM) by Fridrich and Kodovsky (2012)), step two consists of binary classification (an image has a hidden message or not) where are typically used models such as Support Vector Machines (SVM) or perceptrons. In more recent years, thanks to advances in Deep Learning (DL) (Nielsen, 2015) and Graphic Processing Units (GPUs) (Tabares Soto, 2016), DL techniques in steganography and steganalysis have been improving the detection percentages of steganographic images. These techniques are characterized by the unification of feature extraction stage and classification under the same model, reducing complexity and dimensionality introduced by manual feature extraction (Fridrich and Kodovsky, 2012).

Qian et al. (2015) designed the first Convolutional Neural Network (CNN) with a supervised learning approach. This CNN consisted of convolutional layers and used Gaussian Activation. Compared to the detection percentages of state-of-the-art approaches; the results were approximately $4 \%$ lower than those obtained by Spatial Rich Models (SRM) Fridrich and Kodovsky (2012), and approximately 10\% higher than those obtained by Subtractive Pixel Adjacency Matrix (SPAM) Pevny et al. (2010a).

Xu et al. (2016b) proposed an architecture with convolutional layers, similar to Qian et al. (2015). The researchers improved the detection percentages by using an absolute value (ABS) layer and $1 \times 1$ convolutional kernels and modifying the training scheme using CNN as a Base Learner (Xu et al., 2016a) train sets of CNN's, obtaining better training parameters. In the same year, Qian et al. (2016) used Transfer Learning, for the first time in the field, by taking the trained parameters of a CNN with high payload steganographic images and using them to detect images with a low payload. At this point, DL techniques still would not surpass SRM nor SPAM.

Ye et al. (2017) presented a new CNN with eight convolutional layers, Truncation Linear Unit (TLU) as activation function, and filter banks initialized with SRM-based weights for image pre-processing. By mimicking the SRM feature extraction process, the detection results were improved by approximately $10 \%$ compared to traditional algorithms.

Yedroudj et al. (2018) proposed a new CNN. This CNN joined different concepts that were useful in previous designs: filter banks based on SRM, five convolutional layers for feature extraction, Batch Normalization (BN), TLU activation units, and a more significant training database, achieved by adding images from the BOWS 2 database (Mazurczyk and Wendzel, 2017) to the traditional BOSSBase database (Bas et al., 2011), and also by crop, resize, rotate and interpolate operations. With this new architecture, the detection results were improved by approximately $6 \%$ compared to Ye et al. (2017). In the same year, Boroumand et al. (2018) proposed a CNN architecture that detects steganographic images in the spatial and frequency domains. This new architecture used filter banks based on SRM optimized during training, as well as a shortcut or residual connections to allow training such a deep network.

Zhang et al. (2019) proposed a new architecture. This CNN uses an SRM-inspired filter bank in the pre-processing layer weights, separable convolutions, and multi-level average pooling known as Spatial Pyramid Pooling (SPP) (He et al., 2014) to allow the network to analyze arbitrarily sized images.

The most recent contribution was published by Reinel et al. (2021). This network maintains, for the preprocessing stage, the $30 \mathrm{SRM}$ filters and has a $3 \times \mathrm{TanH}$ activation function. This $\mathrm{CNN}$ uses shortcuts for feature extraction and separable and depthwise convolutions. Also, the architecture uses the ELU activation function on all feature extraction convolutions. The CNN does not use fully connected layers; the network uses a softmax directly after the global average pooling. Up until now, this CNN achieves the best detection percentage of steganographic images in the spatial domain.

This article presents a thorough experimentation process where different CNN's architectures were tested under various combinations of computational elements and hyper-parameters to determine which of them are relevant in steganalysis, and then design a strategy to improve steganographic image detection accuracy for multiple architectures. The strategy makes modifications in each stage of the network: pre-processing, feature extraction, and classification. The proposed changes improved the accuracy of three steganalysis CNN's from $2 \%$ up to $10 \%$ while reducing the training time to less than 6 hours and enhancing the stability of the networks. Additionally, this approach allows us to adapt image classification architectures (e.g., VGG16 or VGG19) to the steganalysis application. It is noteworthy the SRM filters for pre-process information and improves accuracy, as well as Spatial Dropout for training stability. Still, all layers are essential to assess the relevance of different aspects of DL algorithms applied to steganalysis, ultimately helping to understand the limitations and approach them. 
The rest of the paper has the following order: Materials and Methods describes the database, computational elements involved in the strategy, and the CNN's architectures engaged in the experiments. Results present the results found. Discussion analyses and discusses the results. At last, Conclusions presents the conclusions of the paper.

\section{MATERIALS AND METHODS}

\section{Databases}

The databases used for the experiments were Break Our Steganographic System (BOSSBase 1.01) (Bas et al., 2011) and Break Our Watermarking System (BOWS 2) (Mazurczyk and Wendzel, 2017). These databases are frequently applied for steganalysis in the spatial domain. Each database has 10,000 cover images in a Portable Gray Map (PGM) format, $512 \times 512$ pixels, and bits in grayscale. BOSSBase and BOWS 2 have similar features and capture devices to avoid the Cover-Source Mismatch effect (Kodovský et al., 2014; Pibre et al., 2016; Chen et al., 2017). For this research, we established a baseline for all the experiments, the following operations were performed on the images:

- All images were resized to $256 \times 256$ pixels.

- Each corresponding steganographic image was created for each cover image using two different algorithms, two payloads of 0.2 bits per pixel (bpp) and $0.4 \mathrm{bpp}$.

- The images were divided into training, validation, and test sets, creating two databases. One with images from BOSSBase 1.01 and the other combining BOSSBase 1.01 and BOWS 2.

- Each set was saved in NumPy array (npy) format, which decreases reading time from 16 to 20 times.

\section{Partition}

We used two database for the experiments, BOSSBase 1.01 and BOSSBase $1.01+$ BOWS 2. The BOSSBase 1.01 database contains 10,000 pairs of images (cover and stego) divided into 4000 pairs for training, 1000 pairs for validation, and 5000 for testing. The partition of the BOSSBase 1.01 database was based on the works by Xu et al. (2016b), Ye et al. (2017) and Zhang et al. (2019).

The BOSSBase 1.01 + BOWS 2 database contains 20,000 pairs of images, divided into 14,000 pairs for training (10,000 BOWS $2+4000$ BOSSBase 1.01), 1000 pairs for validation (BOSSBase 1.01) and 5000 for testing (BOSSBase 1.01). The distribution and partition for this database was done as proposed by Ye et al. (2017), Yedroudj et al. (2018) and Zhang et al. (2019).

\section{Steganographic algorithms}

Two steganographic algorithms were used to embed noise in the cover images from the databases; these were: S-UNIWARD by Holub et al. (2014) and WOW by Holub and Fridrich (2012) with two payloads $(0.2$ and $0.4 \mathrm{bpp})$. The steganographic algorithms based implementation was on the open-source tool named Aletheia (Lerch, 2020) and open-source implementation by Digital Data Embedding Laboratory at Binghamton University (BinghamtonUniversity, 2015).

\section{Computational elements} SRM filter banks

SRM filters were designed by Fridrich and Kodovsky (2012) to enhance and extract steganographic noise from images. These filters were designed and used in steganalysis before introducing CNN's to the field, but as shown by Ye et al. (2017) and Yedroudj et al. (2018), using these filters to initialize the kernels of a convolutional layer improves detection results. Inspired by these works, the preprocessing block uses 30 high-pass filters from the SRM before the feature extraction stage, the selected filters are presented on Figure 1. It is important to note that the convolution kernels' size was set to $5 \times 5$ and to achieve that, some of the filters were padded with zero. 


\begin{tabular}{|c|c|c|c|c|c|c|c|c|c|c|c|c|c|c|c|c|c|c|c|c|c|c|c|c|c|c|c|c|c|}
\hline \multicolumn{20}{|c|}{ Class 1} & \multicolumn{10}{|c|}{ Edge $3 \times 3$} \\
\hline 0 & 0 & 0 & 0 & 0 & 0 & 0 & 0 & 0 & 0 & 0 & 0 & 0 & 0 & 0 & 0 & 0 & 0 & 0 & 0 & 0 & 0 & 0 & 0 & 0 & 0 & 0 & 0 & 0 & 0 \\
\hline 0 & 1 & 0 & 0 & 0 & 0 & 0 & 1 & 0 & 0 & 0 & 0 & 0 & 1 & 0 & 0 & 0 & 0 & 0 & 0 & 0 & -1 & 2 & -1 & 0 & 0 & 0 & 2 & -1 & 0 \\
\hline 0 & 0 & 0 & 0 & 0 & 0 & 0 & 0 & 0 & 0 & 0 & 0 & 0 & 0 & 0 & 0 & 0 & 0 & 0 & 0 & 0 & 0 & 0 & 0 & 0 & 0 & 0 & 2 & -1 & 0 \\
\hline 0 & 0 & 0 & 0 & 0 & 0 & 0 & 0 & 0 & 0 & 0 & 0 & 0 & 0 & 0 & 0 & 0 & 0 & 0 & 0 & 0 & 0 & 0 & 0 & 0 & 0 & 0 & 0 & 0 & 0 \\
\hline 0 & 0 & 0 & 0 & 0 & 0 & 0 & 0 & 0 & 0 & 0 & 0 & 0 & 0 & 0 & 0 & 0 & 0 & 0 & 0 & 0 & 0 & 0 & 0 & 0 & 0 & -1 & 2 & 0 & 0 \\
\hline 0 & 0 & -1 & 1 & 0 & 0 & 0 & -1 & 0 & 0 & 0 & 0 & -1 & 0 & 0 & 0 & 0 & -1 & 0 & 0 & 0 & 2 & -4 & 2 & 0 & 0 & 2 & -4 & 0 & 0 \\
\hline 0 & 0 & 0 & 0 & 0 & 0 & 1 & 0 & 0 & 0 & 0 & 0 & 1 & 0 & 0 & 0 & 0 & 0 & 1 & 0 & 0 & -1 & 2 & -1 & 0 & 0 & -1 & 2 & 0 & 0 \\
\hline \multicolumn{20}{|c|}{ Class 2} & \multicolumn{10}{|c|}{ Edge $5 \times 5$} \\
\hline 0 & 0 & 0 & 0 & 0 & 0 & 0 & 0 & 0 & 0 & 0 & 0 & 0 & 0 & 0 & 0 & 0 & 0 & 0 & 0 & -1 & 2 & -2 & 2 & -1 & 0 & 0 & -2 & 2 & -1 \\
\hline 0 & 1 & 0 & 0 & 0 & 0 & 0 & 1 & 0 & 0 & 0 & 0 & 0 & 1 & 0 & 0 & 0 & 0 & 0 & 0 & 2 & -6 & 8 & -6 & 2 & 0 & 0 & 8 & -6 & 2 \\
\hline 0 & 0 & -2 & 0 & 0 & 0 & 0 & -2 & 0 & 0 & 0 & 0 & -2 & 0 & 0 & 0 & 1 & -2 & 1 & 0 & -2 & 8 & -12 & 8 & -2 & 0 & 0 & -12 & 8 & -2 \\
\hline 0 & 0 & 0 & 1 & 0 & 0 & 0 & 1 & 0 & 0 & 0 & 1 & 0 & 0 & 0 & 0 & 0 & 0 & 0 & 0 & 0 & 0 & 0 & 0 & 0 & 0 & 0 & 8 & -6 & 2 \\
\hline 0 & 0 & 0 & 0 & 0 & 0 & 0 & 0 & 0 & 0 & 0 & 0 & 0 & 0 & 0 & 0 & 0 & 0 & 0 & 0 & 0 & 0 & 0 & 0 & 0 & 0 & 0 & -2 & 2 & -1 \\
\hline \multicolumn{20}{|c|}{ Class 3} & 0 & 0 & 0 & 0 & 0 & -1 & 2 & -2 & 0 & 0 \\
\hline-1 & 0 & 0 & 0 & 0 & 0 & 0 & -1 & 0 & 0 & 0 & 0 & 0 & 0 & -1 & 0 & 0 & 0 & 0 & 0 & 0 & 0 & 0 & O & 0 & ? & 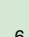 & 8 & 0 & 0 \\
\hline 0 & 3 & 0 & 0 & 0 & 0 & 0 & 3 & 0 & 0 & 0 & 0 & 0 & 3 & 0 & 0 & 0 & 0 & 0 & 0 & & & & & & & & & & \\
\hline 0 & 0 & -3 & 0 & 0 & 0 & 0 & -3 & 0 & 0 & 0 & 0 & -3 & 0 & 0 & 0 & 1 & -3 & 3 & -1 & -2 & 8 & -12 & 8 & -2 & -2 & 8 & -12 & 0 & 0 \\
\hline & & & & & & & & & & & & & & & & & & & & 2 & -6 & 8 & -6 & 2 & 2 & -6 & 8 & 0 & 0 \\
\hline 0 & 0 & 0 & 1 & 0 & 0 & 0 & 1 & 0 & 0 & 0 & 1 & 0 & 0 & 0 & 0 & 0 & 0 & 0 & 0 & -1 & 2 & -2 & 2 & -1 & -1 & 2 & -2 & 0 & 0 \\
\hline 0 & 0 & 0 & 0 & 0 & 0 & 0 & 0 & 0 & 0 & 0 & 0 & 0 & 0 & 0 & 0 & 0 & 0 & 0 & 0 & \multicolumn{5}{|c|}{ square $3 \times 3$} & \multicolumn{5}{|c|}{ square $5 \times 5$} \\
\hline 0 & 0 & 0 & 0 & 0 & 0 & 0 & 0 & 0 & 0 & 0 & 0 & 0 & 0 & 0 & 0 & 0 & 0 & 0 & 0 & 0 & 0 & 0 & 0 & 0 & -1 & 2 & -2 & 2 & -1 \\
\hline 0 & 1 & 0 & 0 & 0 & 0 & 0 & 1 & 0 & 0 & 0 & 0 & 0 & 1 & 0 & 0 & 0 & 0 & 0 & 0 & 0 & -1 & 2 & -1 & 0 & 2 & -6 & 8 & -6 & 2 \\
\hline 0 & 0 & -3 & 0 & 0 & 0 & 0 & -3 & 0 & 0 & 0 & 0 & -3 & 0 & 0 & -1 & 3 & -3 & 1 & 0 & 0 & 2 & -4 & 2 & 0 & -2 & 8 & -12 & 8 & -2 \\
\hline 0 & 0 & 0 & 3 & 0 & 0 & 0 & 3 & 0 & 0 & 0 & 3 & 0 & 0 & 0 & 0 & 0 & 0 & 0 & 0 & 0 & -1 & 2 & -1 & 0 & 2 & -6 & 8 & -6 & 2 \\
\hline 0 & 0 & 0 & 0 & -1 & 0 & 0 & -1 & 0 & 0 & -1 & 0 & 0 & 0 & 0 & 0 & 0 & 0 & 0 & 0 & 0 & 0 & 0 & 0 & 0 & -1 & 2 & -2 & 2 & -1 \\
\hline
\end{tabular}

Figure 1. SRM filters per categories

\section{Batch Normalization (BN)}

BN consists of normalizing each feature distribution, making the average zero and the variance unitary, which results in less sensitive training to the initialization of parameters. This operation allows scaling and translating the distribution, if necessary (Ioffe and Szegedy, 2015). In practice, BN allows for a higher learning rate and improves detection accuracy (Chen et al., 2017). Equation 1 describes the BN used in this study.

Given a random variable $X$ whose realization is a value $x \in \mathbb{R}$ of the feature map, the BN of this value $x$ (Reinel et al., 2019; Tabares-Soto et al., 2020) is:

$$
B N(x, \gamma, \beta)=\beta+\gamma \frac{x-E[X]}{\sqrt{\operatorname{Var}[X]+\varepsilon}}
$$

with $E[X]$ the expectation, $\operatorname{Var}[X]$ the variance, and $\gamma$ and $\beta$ two scalars represent a re-scaling and a re-translation. The expectation and the variance are computed per batch, while $\gamma$ and $\beta$ are optimized during training.

\section{Absolute Value (ABS) layer}

An ABS layer computes the absolute value of the feature maps. When applied in steganalysis, it forces the statistical modeling to take the symmetry of noise residuals into account (Xu et al., 2016b).

\section{Spatial Dropout}

Spatial Dropout was introduced by Tompson et al. (2015) as a type of Dropout for CNN, which improves generalization and reduces overfitting. Compared to traditional Dropout, which "drops" the neuron's 
activation, Spatial Dropout "drops" the entire feature map.

\section{Truncated Linear Unit (TLU) activation function}

The TLU activation function was first introduced by Ye et al. (2017) as a steganalysis particular activation function. This function's motivation is to capture the external signal to noise ratio characteristic of the steganographic embedding procedure, which in general, embeds signals with a much lower amplitude than image content. TLU is a modification of the joint Rectified Linear Unit (ReLU), which performs linear activation, but is truncated at threshold $T$. TLU is defined in Equation 2.

$$
T L U(x)= \begin{cases}-T & \text { if } x<-T \\ x & \text { if }-T \leq x \leq T \\ T & \text { if } x>T\end{cases}
$$

\section{Leaky Rectified Linear Unit (ReLU) activation function}

Leaky ReLU is another modification of the ReLU activation function. In this case, for negative values, the function decreases linearly controlled by the negative slope $m$. This slight modification is useful for specific applications, given it avoids the potential problem of a neuron's output always being zero, the Leaky ReLU is less sensitive to weight initialization and data normalization. Equation 3 defines this activation function.

$$
\operatorname{LeakyReLU}(x)= \begin{cases}m x & \text { if } x<0 \\ x & \text { if } x \geq 0\end{cases}
$$

\section{Hyperbolic Tangent (TanH) activation function}

TanH activation function is commonly used in neural networks. It provides nonlinear activation while being a smooth differentiable function. TanH range is also constrained. Equation $\mathbf{4}$ defines TanH.

$$
\operatorname{Tan} H(x)=\frac{e^{x}-e^{-x}}{e^{x}+e^{-x}}
$$

\section{CNN Architectures}

The strategy was developed and tested on three CNN architectures designed for steganalysis in the spatial domain and two image classification CNN architectures.

\section{Xu-Net}

$\mathrm{Xu}-\mathrm{Net}$ is the name for the CNN proposed by Xu et al. (2016b). This architecture has a feature extraction stage composed of a High Pass Filter (HPF) layer, five convolutional layers for feature extraction, an ABS layer after the first convolutional layer, BN after each convolutional layer, a classification stage that consists of two fully connected layers, and one Softmax. The first two layers use the TanH activation function and ReLU for the last three layers (Reinel et al., 2019).

The mini-batch gradient descent optimizer was used for the training process, with momentum fixed to 0.9 , and the learning rate initialized to 0.001 , scheduled to decrease $10 \%$ every 5000 iterations. Each mini-batch consisted of 64 images ( 32 cover/stego pairs). The CNN was trained for 120,000 iterations.

\section{Ye-Net}

This network proposed by Ye et al. (2017), it uses an SRM filter bank for steganographic noise extraction. The feature extraction stage consists of eight convolutional layers, a TLU activation function after the first layer, and TanH for the others. The classification stage has one fully connected layer and Softmax activation function.

In the original Ye-Net work, the AdaDelta optimizer was used, with momentum fixed to 0.95, weight decay set to $5 \times 10^{-4}$, the "delta" parameter was $1 \times 10^{-8}$, and the learning rate initialized to 0.4 . Each mini-batch consisted of 32 images ( 16 cover/stego pairs). The CNN was trained for different number of epochs based on the experiment and behavior of accuracy. 


\section{Yedroudj-Net}

This network proposed by Yedroudj et al. (2018), it takes the best features of the Xu-Net, and Ye-Net and unifies them under the same architecture. This architecture uses an SRM-inspired filter bank, five convolutional layers for feature extraction, an ABS layer after the first one, Average Pooling after each layer, starting from the second one. It uses the TLU activation function in the first two layers and ReLU in the last three layers. The classification stage has two fully connected layers and Softmax activation function.

They applied a mini-batch stochastic gradient descent (SGD) optimizer. The momentum was fixed to 0.95 and the weight decay to 0.0001 . The learning rate (initialized to 0.01 ) was decreased by a factor of 0.1 , each $10 \%$ of the total number of epochs. The mini-batch size was set to 16 ( 8 cover/stego pairs), due to GPU memory limitation.

\section{VGG16 and VGG19}

VGG16 and VGG19 are CNN's proposed by Simonyan and Zisserman (2015). These architectures were initially designed for image classification and presented for the Large Scale Visual Recognition Challenge 2014 (StanfordVisionLab, 2014), achieving 93.2\% top-5 test accuracy in ImageNet. The number in the network name represents the number of weight layers each architecture has; VGG16 has 16 weight layers (13 convolutional and three fully connected layers). VGG19 has 19 weight layers (16 convolutional and three fully connected layers). Both architectures consist of 5 convolutional blocks (variable number of convolutional layers), each followed by Max Pooling, three fully connected layers, and Softmax activation function at the end for classification purposes. All hidden layers use the ReLU activation function.

\section{Strategy}

With all the computational elements mentioned before, we transform all architectures by the following changes:

- Input image resized to $256 \times 256$

- All SRM filters were applied in the preprocessing block by a convolution, followed by a $3 \times$ Tan $H$ activation, which is a modified TanH with range $(-3,3)$.

- Spatial Dropout applied in Convolutional blocks beginning with the second one.

- Activation use in Convolutional blocks were Leaky ReLU.

- Add Absolute layer (ABS) after activation in Convolutional blocks.

- Batch Normalization layer (BN) after the absolute layer in Convolutional blocks.

- Concatenation layer with triple input of the last layer, located after the first and last BN.

- The classification stage, shown in Figure 2, consists of three fully connected layers (128, 64 and 32 units, respectively) with Leaky ReLU activation and Softmax activation function. This stage is located after the global average pooling layer and is the same in all architectures.

- The optimizer was stochastic gradient descent.

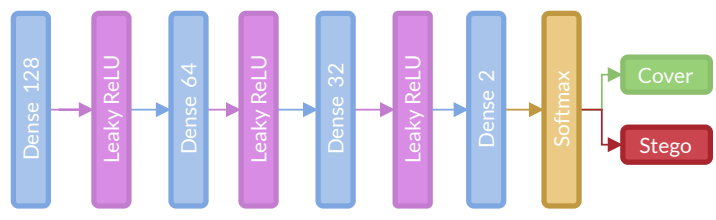

Figure 2. Classification stage implemented for the strategy 
Figure 3 presents the changes in Ye-Net Architecture as an example of how the strategy is applied, and Figure 4 shows strategy applied over classification models (Especificly VGG16)

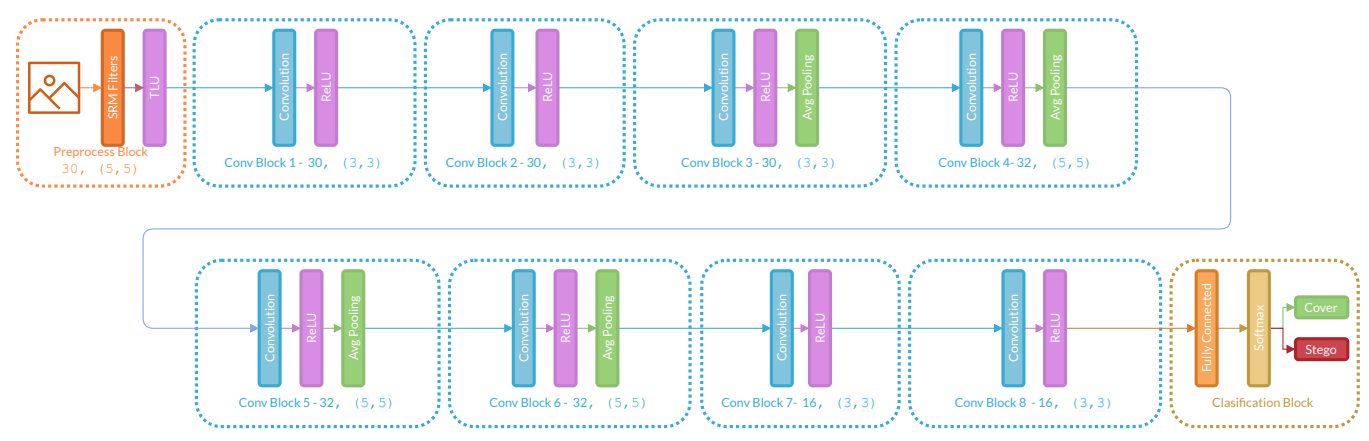

A

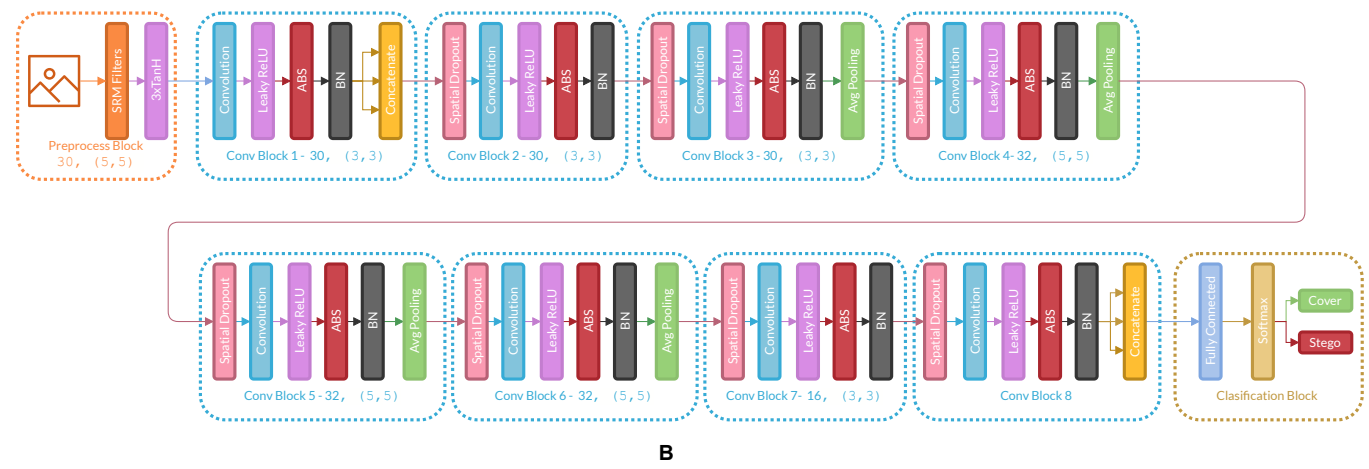

Figure 3. A. Architecture of the original Ye-Net, B. Ye-Net architecture with the strategy applied. 

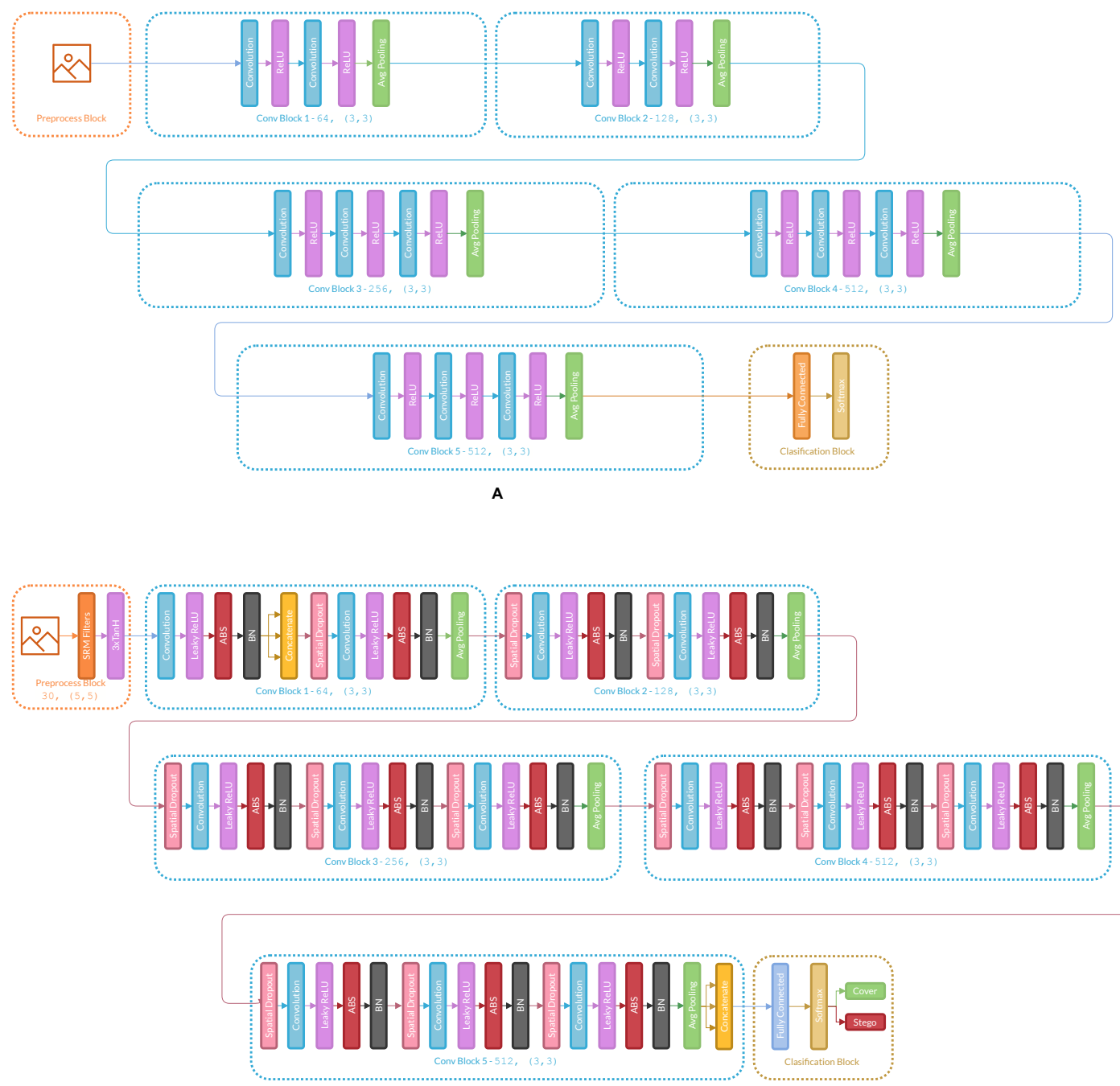

B

Figure 4. A. Architecture of the original VGG16, B. VGG16 architecture with the strategy applied.

\section{Hyper-parameters}

Convolutional and fully connected layers weights have a glorot normal initializer and use L2 regularization for kernels and bias. The spatial dropout rate has an 0.1 value. BN has a momentum of 0.2 , epsilon of 0.001 , and renorm momentum of 0.4 value. The stochastic gradient descent optimizer momentum is 0.95 , and a learning rate initialized to 0.005 . Finally, activation in convolutional layers was a modified ReLU with a negative slope of 0.1 , converting a ReLU into a Leaky ReLU.

\section{Training}

The batch size is set to 64 images for the steganalysis CNN's (Xu-Net, Ye-Net, and Yedroudj-Net) and 32 images for the VGG16 and VGG19 due to their bigger network size. The number of epochs needed to train the architectures varies depending on the database, payload, and model. Ye-Net and Yedroudj-Net are trained for 100 epochs in both databases with $0.4 \mathrm{bpp}$, while Xu-Net was trained 150 epochs. VGG16 and VGG19 in BOSSBase 1.01 with 0.4 bpp are trained for 100 and 160 epochs, respectively; in BOSSBase $1.01+$ BOWS 2 with 0.4 bpp, only 60 epochs are necessary for convergence in both networks. To train the networks with $0.2 \mathrm{bpp}$, their weights were initialized with the weights obtained from the model trained with 0.4 bpp (transfer learning). For 0.2 bpp, all CNN's were trained for 50 epochs. 
Table 1. Accuracy in test S-UNIWARD stego-images with different payloads using BOSSBase 1.01 and BOSSBase $1.01+$ BOWS 2

\begin{tabular}{|l|c|c|c|c|c|c|c|c|}
\hline Dataset & \multicolumn{4}{|c|}{ BOSSBase 1.01 } & \multicolumn{3}{c|}{ BOSSBase 1.01 + BOWS 2 } \\
\hline Results & Reported in literature & \multicolumn{2}{|c|}{ Strategy } & \multicolumn{2}{c|}{ Reported in literature } & \multicolumn{3}{c|}{ Strategy } \\
\hline Payload & 0.2 bpp & 0.4 bpp & 0.2 bpp & $0.4 \mathrm{bpp}$ & 0.2 bpp & 0.4 bpp & 0.2 bpp & $0.4 \mathrm{bpp}$ \\
\hline Xu-Net & 0.6090 & 0.7280 & $\mathbf{0 . 6 8 2 9}$ & $\mathbf{0 . 7 8 1 9}$ & - & - & $\mathbf{0 . 7 1 2 1}$ & $\mathbf{0 . 8 1 8 2}$ \\
Ye-Net & 0.6000 & 0.6880 & $\mathbf{0 . 7 1 0 3}$ & $\mathbf{0 . 8 1 0 1}$ & - & - & $\mathbf{0 . 7 2 6 9}$ & $\mathbf{0 . 8 3 3 8}$ \\
Yedroudj-Net & 0.6330 & 0.7720 & $\mathbf{0 . 6 7 7 3}$ & $\mathbf{0 . 7 9 6 4}$ & 0.6560 & - & $\mathbf{0 . 7 3 3 5}$ & $\mathbf{0 . 8 4 1 5}$ \\
\hline
\end{tabular}


Table 2. Accuracy in test WOW stego-images with different payloads using BOSSBase 1.01 and BOSSBase $1.01+$ BOWS 2

\begin{tabular}{|l|c|c|c|c|c|c|c|c|}
\hline Dataset & \multicolumn{4}{|c|}{ BOSSBase 1.01} & \multicolumn{3}{c|}{ BOSSBase 1.01 + BOWS 2 } \\
\hline Results & Reported in literature & \multicolumn{2}{|c|}{ Strategy } & Reported in literature & \multicolumn{3}{c|}{ Strategy } \\
\hline Payload & 0.2 bpp & 0.4 bpp & 0.2 bpp & $0.4 \mathrm{bpp}$ & 0.2 bpp & 0.4 bpp & 0.2 bpp & $0.4 \mathrm{bpp}$ \\
\hline Xu-Net & 0.6760 & 0.7930 & $\mathbf{0 . 7 3 5 2}$ & $\mathbf{0 . 8 2 2 1}$ & - & - & $\mathbf{0 . 7 4 8 3}$ & $\mathbf{0 . 8 4 7 6}$ \\
Ye-Net & 0.6690 & 0.7680 & $\mathbf{0 . 7 5 4 7}$ & $\mathbf{0 . 8 4 5 1}$ & 0.7390 & - & $\mathbf{0 . 7 7 1 3}$ & $\mathbf{0 . 8 6 2 3}$ \\
Yedroudj-Net & 0.7220 & 0.8590 & $\mathbf{0 . 7 6 2 3}$ & $\mathbf{0 . 8 4 7 0}$ & 0.7630 & - & $\mathbf{0 . 7 8 2 2}$ & $\mathbf{0 . 8 6 9 1}$ \\
\hline
\end{tabular}

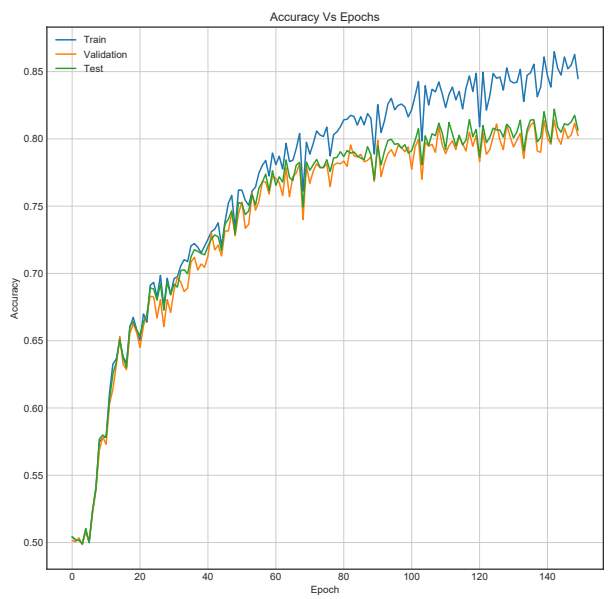

A

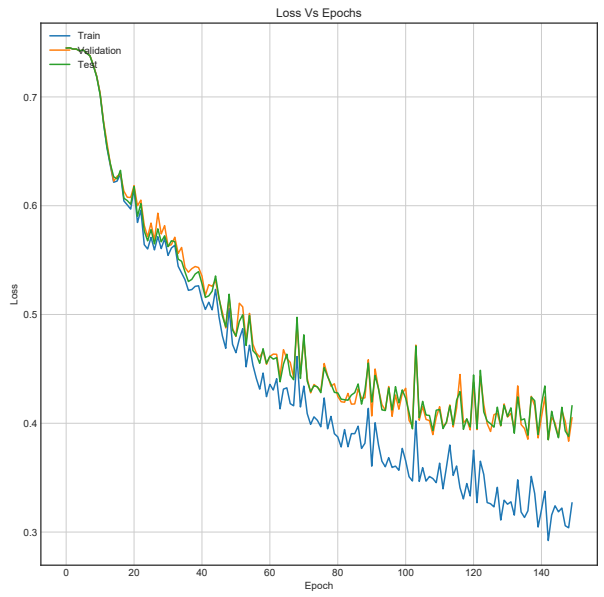

B

Figure 6. Training curves of Xu-Net with BOSSBase 1.01 WOW 0.4 bpp. A. Accuracy, B. Loss.

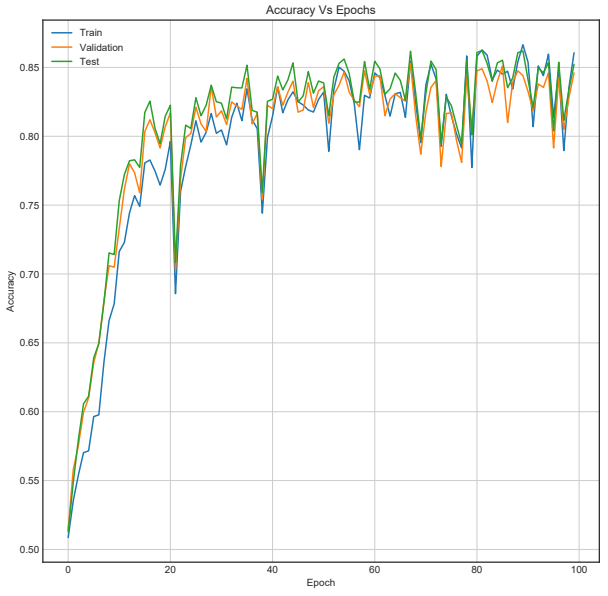

A

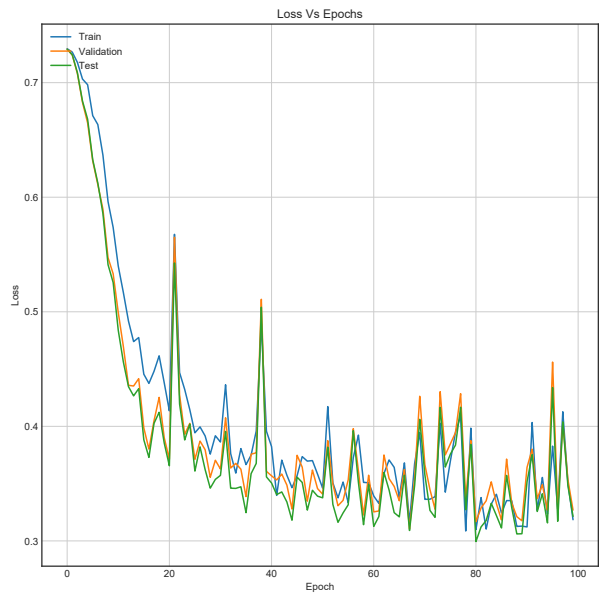

B

Figure 7. Training curves of Ye-Net with BOSSBase 1.01 + BOWS 2 WOW 0.4 bpp. A. Accuracy, B. Loss. 


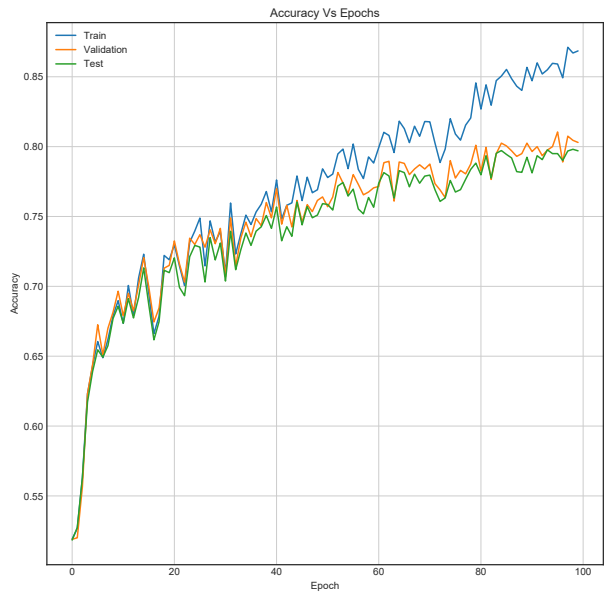

A

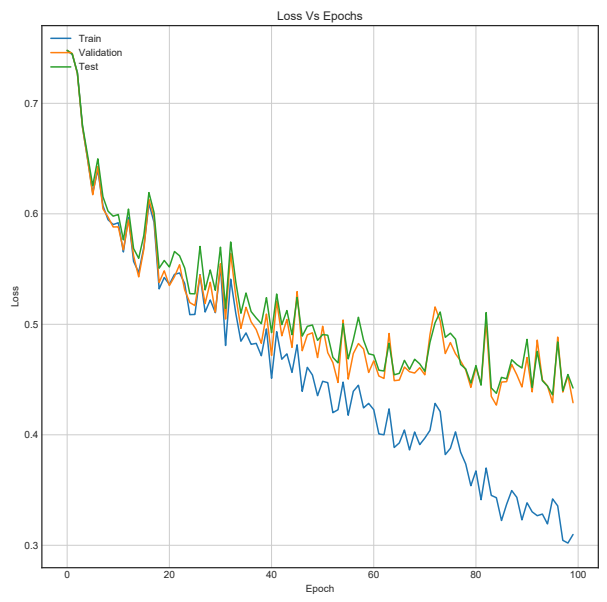

B

Figure 8. Training curves of Yedroudj-Net with BOSSBase 1.01 S-UNIWARD 0.4 bpp. A. Accuracy, B. Loss.

Table 3. Accuracy in test S-UNIWARD stego-images with VGG16Stego-VGG19Stego and different payloads using BOSSBase 1.01 and BOSSBase 1.01 + BOWS 2

\begin{tabular}{|l|c|c|c|c|c|c|c|c|}
\hline Dataset & \multicolumn{4}{|c|}{ BOSSBase 1.01 } & \multicolumn{3}{c|}{ BOSSBase 1.01 + BOWS 2 } \\
\hline Pooling & \multicolumn{2}{|c|}{ Max Pooling } & \multicolumn{2}{|c|}{ Average Pooling } & \multicolumn{3}{c|}{ Max Pooling } & \multicolumn{2}{c|}{ Average Pooling } \\
\hline Payload & $0.2 \mathrm{bpp}$ & $0.4 \mathrm{bpp}$ & $0.2 \mathrm{bpp}$ & $0.4 \mathrm{bpp}$ & $0.2 \mathrm{bpp}$ & $0.4 \mathrm{bpp}$ & $0.2 \mathrm{bpp}$ & $0.4 \mathrm{bpp}$ \\
\hline VGG16Stego & 0.7370 & 0.8291 & 0.7356 & 0.8370 & 0.7513 & 0.8545 & 0.7473 & 0.8511 \\
VGG19Stego & 0.7420 & 0.8210 & 0.7417 & 0.8291 & 0.7409 & 0.8520 & 0.7550 & 0.8490 \\
\hline
\end{tabular}

Table 4. Accuracy in test WOW stego-images with VGG16Stego-VGG19Stego and different payloads using BOSSBase 1.01 and BOSSBase 1.01 + BOWS 2

\begin{tabular}{|l|c|c|c|c|c|c|c|c|}
\hline Dataset & \multicolumn{4}{|c|}{ BOSSBase 1.01 } & \multicolumn{3}{c|}{ BOSSBase 1.01 + BOWS 2 } \\
\hline Pooling & \multicolumn{2}{|c|}{ Max Pooling } & \multicolumn{2}{|c|}{ Average Pooling } & \multicolumn{2}{c|}{ Max Pooling } & \multicolumn{2}{c|}{ Average Pooling } \\
\hline Payload & $0.2 \mathrm{bpp}$ & $0.4 \mathrm{bpp}$ & $0.2 \mathrm{bpp}$ & $0.4 \mathrm{bpp}$ & $0.2 \mathrm{bpp}$ & $0.4 \mathrm{bpp}$ & $0.2 \mathrm{bpp}$ & $0.4 \mathrm{bpp}$ \\
\hline VGG16Stego & 0.7760 & 0.8556 & 0.7857 & 0.8640 & 0.8059 & 0.8825 & 0.8017 & 0.8830 \\
VGG19Stego & 0.7820 & 0.8570 & 0.7930 & 0.8656 & 0.8060 & 0.8833 & 0.8055 & 0.8857 \\
\hline
\end{tabular}




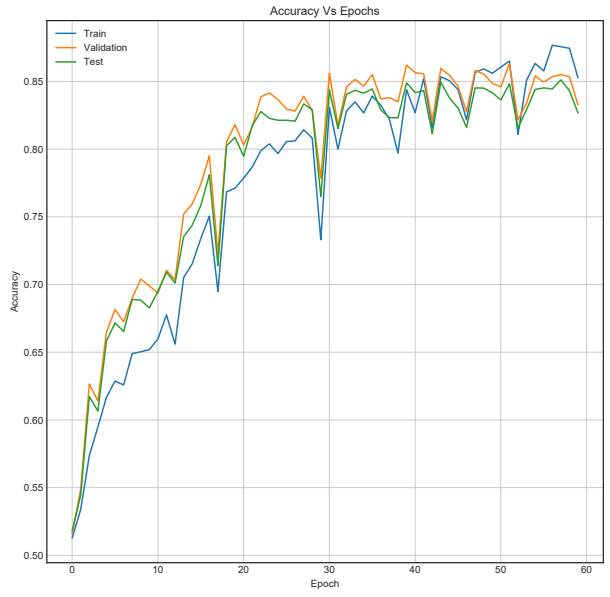

A

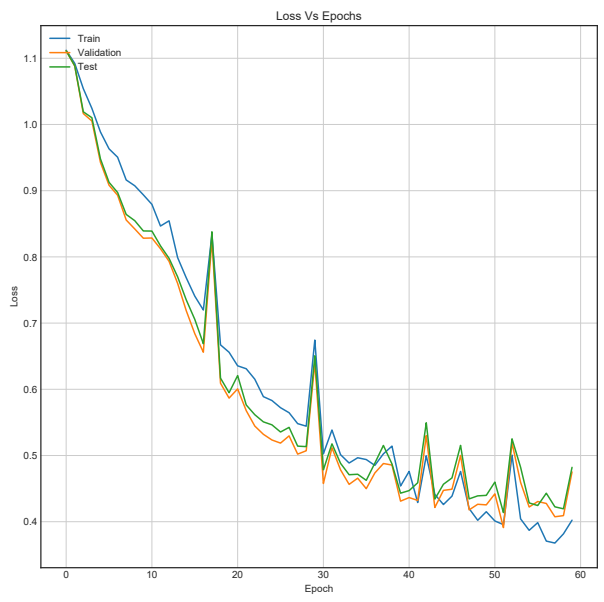

B

Figure 9. Training curves of VGG16Stego Average Pooling with BOSSBase $1.01+$ BOWS 2 S-UNIWARD 0.4 bpp. A. Accuracy, B. Loss.
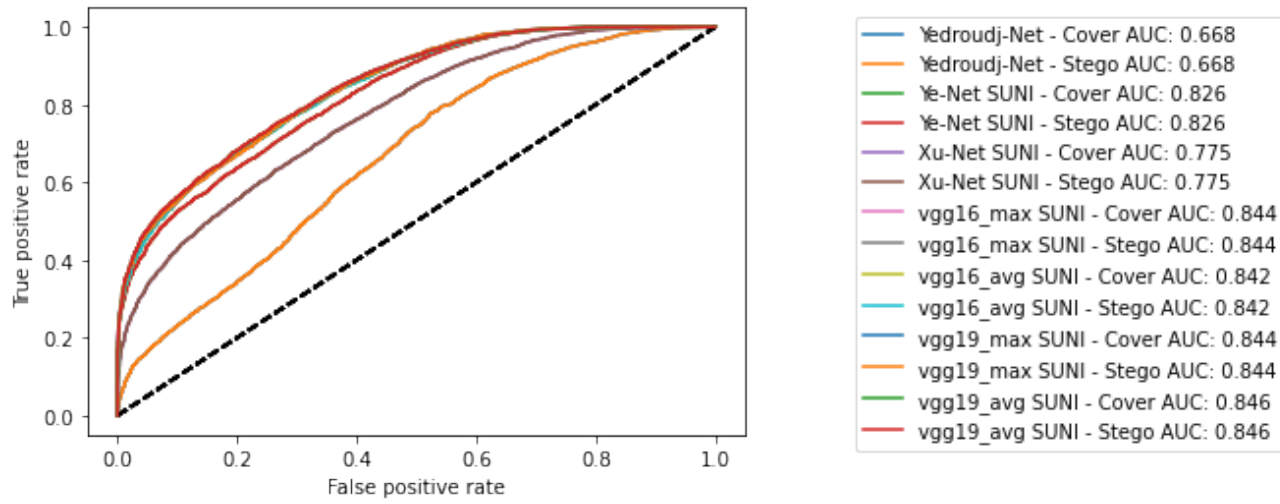

A

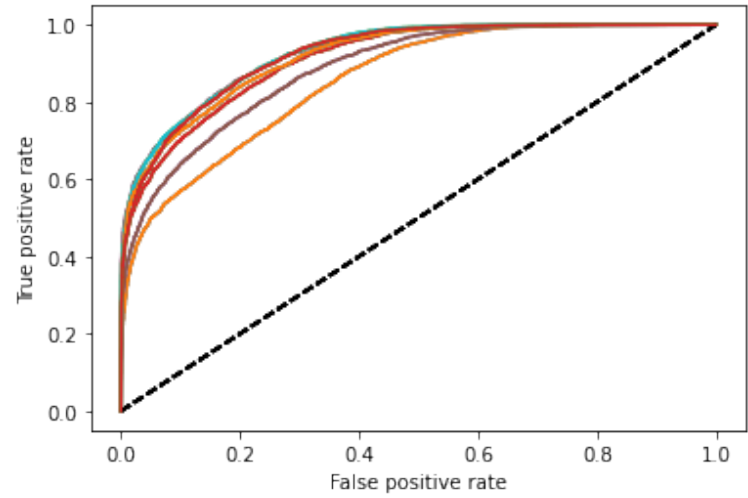

kedroudj-Net SUNI - Cover AUC: 0.856 \edroudj-Net SUNI - Stego AUC: 0.856

ץ-Net SUNI - Cover AUC: 0.912

ie-Net SUNI - Stego AUC: 0.912

Xu-Net SUNI - Cover AUC: 0.886

Xu-Net SUNI - Stego AUC: 0.886

vgg16 max SUNI - Cover AUC: 0.928

_ vgg16_max SUNI - Stego AUC: 0.928

- vgg16_avg SUNI - Cover AUC: 0.928

_ vgg16_avg SUNI - Stego AUC: 0.928

vgg19 max SUNI - Cover AUC: 0.919

- vgg19 max SUNI - Stego AUC: 0.919

- vgg19_avg SUNI - Cover AUC: 0.924

— vgg19_avg SUNI - Stego AUC: 0.924

B

Figure 10. ROC Test curves of all experiment in S-UNIWARD. A. payload of $0.2 \mathrm{bpp}$, B.payload of 0.4 bpp. 

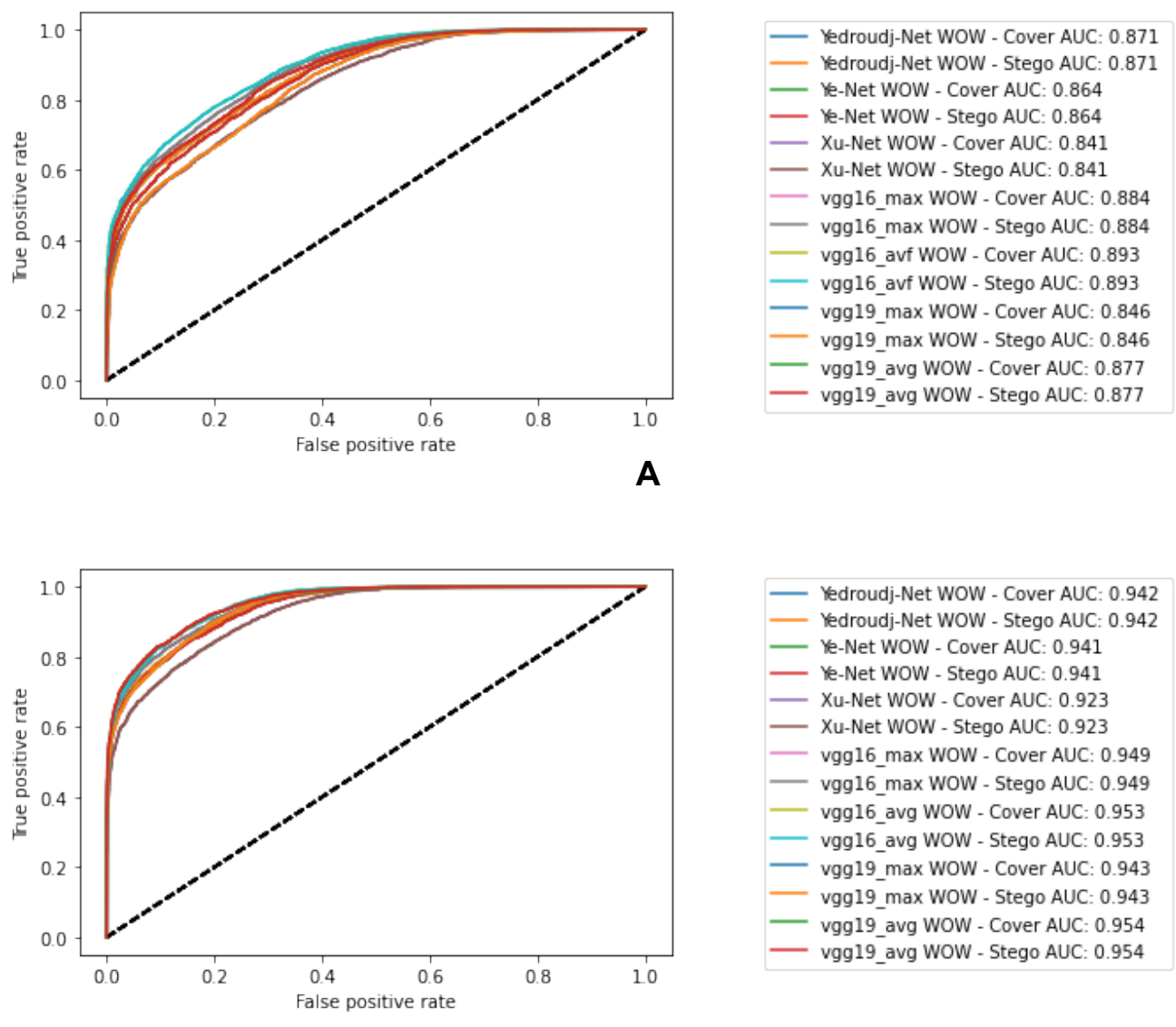

Figure 11. ROC Test curves of all experiments in WOW. A. payload of $0.2 \mathrm{bpp}$, B.payload of $0.4 \mathrm{bpp}$.

\section{DISCUSSION}

This study presents the results of testing different combinations of computational elements and hyperparameters of CNN architectures applied to image steganalysis in the spatial domain, which led to identifying relevant elements for this task and the designing a general strategy to improve the CNN's. There were improvements in the convergence and stability of the training process and steganographic images' detection accuracy.

Regarding detection accuracy, the steganalysis CNN's (Xu-Net, Ye-Net, and Yedroudj-Net) perceived an improvement from $2 \%$ up to $10 \%$ in both steganographic algorithms and payloads. This performance boost can be attributed to the preprocessing stage involving the SRM filter bank and the modified $3 \times$ TanH activation function. The SRM filter bank objective is to enhance steganographic noise in images, and as proven before, it improves detection accuracy (Ye et al., 2017; Yedroudj et al., 2018). TLU function inspired the activation function. As shown by Ye et al. (2017), it is better at capturing the steganographic noise than other activation functions, and the threshold value that yielded the best results was $T=3$. Both TLU and TanH have a similar shape, but the latter is a smooth differentiable function, and the amplification by 3 mimics the desired behavior of the TLU function. On the other hand, the VGG16 and VGG19 image classification CNN's did not surpass the 0.5 detection accuracy before applying the strategy. In contrast, with the strategy, the results overcome those achieved by the steganalysis CNN's. From the results presented in Table 3 and Table 4, it is important to note that, in most cases, the results with Average Pooling are better than those achieved with Max Pooling. In general, Average Pooling is preferred in steganalysis applications because it preserves the steganographic noise better than Max Pooling Qian et al. (2015), given its low amplitude compared to image content. Additionally, the three-layer classification stage provides deeper processing of the features extracted in the convolutional layers, improving detection 
accuracy.

The mentioned improvements in convergence refer to the lower number of epochs and iterations needed to train the CNN's, which means training in less time. In comparison, in the original paper of the Xu-Net (Xu et al., 2016b), it was trained for 120,000 iterations with a mini-batch of size 64; With the strategy, the Xu-Net architecture was trained for 18,750 iterations with the same mini-batch size, while improving the detection accuracy. The training process duration can not be compared because they depend on other factors like hardware specifications. However, it is worth mentioning that it did not take longer than 10 hours to train image classification CNN's and less than 6 hours to train steganalysis CNN's. Table 5 is the approximated time of each CNN.

Table 5. Approximate time of training in all models

\begin{tabular}{|l|c|c|c|c|}
\hline Dataset & \multicolumn{2}{|c|}{ BOSSBase 1.01 } & \multicolumn{2}{c|}{ BOSSBase 1.01 + BOWS 2 } \\
\hline Payload & $0.2 \mathrm{bpp}$ & $0.4 \mathrm{bpp}$ & $0.2 \mathrm{bpp}$ & $0.4 \mathrm{bpp}$ \\
\hline Xu-Net & $\sim 20 \mathrm{~min}$ & $\sim 60 \mathrm{~min}$ & $\sim 70 \mathrm{~min}$ & $\sim 210 \mathrm{~min}$ \\
\hline Ye-Net & $\sim 80 \mathrm{~min}$ & $\sim 180 \mathrm{~min}$ & $\sim 140 \mathrm{~min}$ & $\sim 280 \mathrm{~min}$ \\
\hline Yedroudj-Net & $\sim 100 \mathrm{~min}$ & $\sim 220 \mathrm{~min}$ & $\sim 350 \mathrm{~min}$ & $\sim 400 \mathrm{~min}$ \\
\hline VGG16Stego & $\sim 180 \mathrm{~min}$ & $\sim 240 \mathrm{~min}$ & $\sim 400 \mathrm{~min}$ & $\sim 460 \mathrm{~min}$ \\
\hline VGG19Stego & $\sim 200 \mathrm{~min}$ & $\sim 310 \mathrm{~min}$ & $\sim 410 \mathrm{~min}$ & $\sim 580 \mathrm{~min}$ \\
\hline
\end{tabular}

Similarly, it is challenging to compare training stability improvement, which refers to less variability on the training curves, given the original papers' lack of training curves. For this purpose, we were able to reproduce the original $\mathrm{Xu}-\mathrm{Net}$ architecture to compare the training curves with and without the strategy. By comparing Figure $\mathbf{6}$ to Figure 5, it is possible to observe how accuracy and loss curves vary less over time. In practice, the computational element found to improve the training stability was the Spatial Dropout. By adding this operation before the convolutional layers, the training curves were smoother, although it also forces add epochs to reach convergence.

\section{CONCLUSIONS}

This work presents a strategy to improve the CNN's applied to image steganalysis in the spatial domain. The strategy's key is combining the following computational elements: SRM filter bank and $3 \times$ TanH activation for the preprocessing stage, Spatial Dropout, Absolute Value layer, Batch Normalization and fully connected. The performance improvement can be seen in the convergence and stability of the training process and the detection accuracy. VGG16Stego and VGG19Stego obtained the best performances. Future work should be aimed to optimize the strategy and test it on recent steganalysis CNN's, SR-Net by Boroumand et al. (2018) and Zhu-Net by Zhang et al. (2019). Additionally, demonstrate experimentally the influence of each layer and hyperparameter added by the strategy.

\section{REFERENCES}

Abadi, M., Agarwal, A., Barham, P., Brevdo, E., Chen, Z., Citro, C., Corrado, G. S., Davis, A., Dean, J., Devin, M., Ghemawat, S., Goodfellow, I., Harp, A., Irving, G., Isard, M., Jia, Y., Jozefowicz, R., Kaiser, L., Kudlur, M., Levenberg, J., Mané, D., Monga, R., Moore, S., Murray, D., Olah, C., Schuster, M., Shlens, J., Steiner, B., Sutskever, I., Talwar, K., Tucker, P., Vanhoucke, V., Vasudevan, V., Viégas, F., Vinyals, O., Warden, P., Wattenberg, M., Wicke, M., Yu, Y., and Zheng, X. (2015). TensorFlow: Large-scale machine learning on heterogeneous systems. Software available from tensorflow.org.

Bas, P., Filler, T., and Pevny, T. (2011). "Break Our Steganographic System": The Ins and Outs of Organizing BOSS. In INFORMATION HIDING, volume 6958/2011 of Lecture Notes in Computer Science, pages 59-70, Czech Republic.

BinghamtonUniversity (2015). Steganographic algorithms. http://dde.binghamton.edu/download/stego_algorithms/. 2020-10-18.

Boroumand, M., Chen, M., and Fridrich, J. (2018). Deep Residual Network for Steganalysis of Digital Images. IEEE Transactions on Information Forensics and Security, 14(5):1181-1193. 
Chen, M., Sedighi, V., Boroumand, M., and Fridrich, J. (2017). JPEG-Phase-Aware Convolutional Neural Network for Steganalysis of JPEG Images. Proceedings of the 5th ACM Workshop on Information Hiding and Multimedia Security - IHMMSec '17, pages 75-84.

Fridrich, J., Goljan, M., and Du, R. (2001). Detecting LSB steganography in color, and gray-scale images. IEEE MultiMedia, 8(4):22-28.

Fridrich, J. and Kodovsky, J. (2012). Rich Models for Steganalysis of Digital Images. IEEE Transactions on Information Forensics and Security, 7(3):868-882.

Guo, L., Ni, J., and Shi, Y.-Q. Q. (2014). Uniform Embedding for Efficient JPEG Steganography. IEEE Transactions on Information Forensics and Security, 9(5):814-825.

Guo, L., Ni, J., Su, W., Tang, C., and Shi, Y.-Q. (2015). Using Statistical Image Model for JPEG Steganography: Uniform Embedding Revisited. IEEE Transactions on Information Forensics and Security, 10(12):2669-2680.

He, K., Zhang, X., Ren, S., and Sun, J. (2014). Spatial Pyramid Pooling in Deep Convolutional Networks for Visual Recognition. CoRR, abs/1406.4:1-14.

Holub, V. and Fridrich, J. (2012). Designing steganographic distortion using directional filters. In 2012 IEEE International Workshop on Information Forensics and Security (WIFS), pages 234-239.

Holub, V., Fridrich, J., and Denemark, T. (2014). Universal distortion function for steganography in an arbitrary domain. EURASIP Journal on Information Security, 2014(1):1.

Ioffe, S. and Szegedy, C. (2015). Batch Normalization: Accelerating Deep Network Training by Reducing Internal Covariate Shift. In Proceedings of the 32Nd International Conference on International Conference on Machine Learning - Volume 37, ICML'15, pages 448-456. JMLR.org.

Johnson, N. F. and Jajodia, S. (1998). Exploring steganography: Seeing the unseen. Computer, 31(2):2634.

Kodovský, J., Sedighi, V., and Fridrich, J. (2014). Study of cover source mismatch in steganalysis and ways to mitigate its impact. In Alattar, A. M., Memon, N. D., and Heitzenrater, C. D., editors, Media Watermarking, Security, and Forensics 2014, volume 9028, pages 204-215. International Society for Optics and Photonics, SPIE.

Lerch, D. (2020). Aletheia. https://daniellerch.me/. 2020-10-10.

Li, B., Wang, M., Huang, J., and Li, X. (2014). A new cost function for spatial image steganography. In 2014 IEEE International Conference on Image Processing (ICIP), pages 4206-4210.

Mazurczyk, W. and Wendzel, S. (2017). Information Hiding: Challenges for Forensic Experts. Commun. ACM, 61(1):86-94.

Nielsen, M. A. (2015). Neural Networks and Deep Learning. Machine Learning, pages 875-936.

Pevny, T., Bas, P., and Fridrich, J. (2010a). Steganalysis by Subtractive Pixel Adjacency Matrix. IEEE Transactions on Information Forensics and Security, 5(2):215-224.

Pevny, T., Filler, T., and Bas, P. (2010b). Using High-Dimensional Image Models to Perform Highly Undetectable Steganography. In Bohme, R., Fong, P. W. L., and Safavi-Naini, R., editors, Information Hiding, pages 161-177, Berlin, Heidelberg. Springer Berlin Heidelberg.

Pibre, L., Jerome, P., Ienco, D., and Chaumont, M. (2016). Deep learning is a good steganalysis tool when embedding key is reused for different images, even if there is a cover source-mismatch. Media Watermarking, Security, and Forensics 2016, San Francisco, California, USA, February 14-18, 2016.

Qian, Y., Dong, J., Wang, W., and Tan, T. (2015). Deep learning for steganalysis via convolutional neural networks. IS\&T International Symposium on Electronic Imaging (EI 2015 ), 9409:94090J.

Qian, Y., Dong, J., Wang, W., and Tan, T. (2016). Learning and transferring representations for image steganalysis using convolutional neural network. 2016 IEEE International Conference on Image Processing (ICIP), pages 2752-2756.

Reinel, T., Raúl, R., and Gustavo, I. (2019). Deep learning applied to steganalysis of digital images: A systematic review. IEEE Access, 7:68970-68990.

Reinel, T. S., Brayan, A. A. H., Alejandro, B. O. M., Alejandro, M. R., Daniel, A. G., Alejandro, A. G. J., Buenaventura, B. J. A., Simon, O. A., Gustavo, I., and Raúl, R. P. (2021). Gbras-net: A convolutional neural network architecture for spatial image steganalysis. IEEE Access, 9:14340-14350.

Sedighi, V., Cogranne, R., Fridrich, J., and Member, S. (2016). Content-Adaptive Steganography by Minimizing Statistical Detectability. IEEE Transactions on Information Forensics and Security, 11(2):221-234.

Simmons, G. J. (1984). The Prisoners' Problem and the Subliminal Channel. In Chaum, D., editor, 
Advances in Cryptology: Proceedings of Crypto 83, pages 51-67. Springer US, Boston, MA.

Simonyan, K. and Zisserman, A. (2015). Very deep convolutional networks for large-scale image recognition.

StanfordVisionLab (2014). Results of large scale visual recognition challenge 2014 (ilsvrc2014). http://www.image-net.org/challenges/LSVRC/2014/results. 2020-10-30.

Tabares Soto, R. (2016). Programación paralela sobre arquitecturas heterogéneas.

Tabares-Soto, R., Ramos-Pollán, R., Isaza, G., Orozco-Arias, S., Ortíz, M. A. B., Arteaga Arteaga, H. B., Rubio, A. M., and Alzate Grisales, J. A. (2020). 12 - Digital media steganalysis. In Hassaballah, M., editor, Digital Media Steganography, pages 259-293. Academic Press.

Tompson, J., Goroshin, R., Jain, A., LeCun, Y., and Bregler, C. (2015). Efficient object localization using convolutional networks.

Westfeld, A. (2001). F5-A Steganographic Algorithm. In Moskowitz, I. S., editor, Information Hiding, pages 289-302, Berlin, Heidelberg. Springer Berlin Heidelberg.

Xu, G., Wu, H.-Z., and Shi, Y. Q. (2016a). Ensemble of CNNs for Steganalysis : An Empirical Study. Proceedings of the 4th ACM Workshop on Information Hiding and Multimedia Security, pages 103-107.

Xu, G., Wu, H.-Z., and Shi, Y.-Q. (2016b). Structural design of convolutional neural networks for steganalysis. IEEE Signal Processing Letters, 23(5):708-712.

Ye, J., Ni, J., and Yi, Y. (2017). Deep Learning Hierarchical Representations for Image Steganalysis. IEEE Transactions on Information Forensics and Security, 12(11):2545-2557.

Yedroudj, M., Comby, F., and Chaumont, M. (2018). Yedrouj-Net: An efficient $\{\mathrm{CNN}\}$ for spatial steganalysis. International Conference on Acoustics, Speech, and Signal Processing, abs/1803.0(April).

Zhang, R., Zhu, F., Liu, J., and Liu, G. (2019). Depth-wise separable convolutions and multi-level pooling for an efficient spatial CNN-based steganalysis. IEEE Transactions on Information Forensics and Security, PP(September):1-1. 